\title{
Searches for Light Dark Matter with the CRESST-III Experiment
}

M. Mancuso ${ }^{1}$ (D) A. H. Abdelhameed ${ }^{1} \cdot$ G. Angloher ${ }^{1} \cdot$ R. Breier ${ }^{2} \cdot$ P. Bauer ${ }^{1}$. A. Bento ${ }^{1,10}$ - E. Bertoldo ${ }^{1}$. C. Bucci ${ }^{3}$ - L. Canonica ${ }^{1}$ - A. D'Addabbo ${ }^{3,11}$. S. Di Lorenzo ${ }^{3,11} \cdot$ A. Erb ${ }^{4,9} \cdot$ F. von Feilitzsch ${ }^{4} \cdot$ N. Ferreiro lachellini ${ }^{1}$. S. Fichtinger ${ }^{6} \cdot$ A. Fuss ${ }^{6,7} \cdot$ P. Gorla ${ }^{3} \cdot$ D. Hauff ${ }^{1} \cdot$ M. Ješkovský ${ }^{2} \cdot$ J. Jochum $^{5}$. J. Kaizer ${ }^{2} \cdot$ A. Kinast ${ }^{4} \cdot$ H. Kluck ${ }^{6,7} \cdot$ H. Kraus ${ }^{8} \cdot$ A. Langenkämper ${ }^{4}$ - V. Mokina ${ }^{6}$. E. Mondragon ${ }^{4} \cdot$ M. Olmi ${ }^{3,11} \cdot$ T. Ortmann ${ }^{4} \cdot$ C. Pagliarone ${ }^{3,13} \cdot$ V. Palušová ${ }^{2}$. L. Pattavina ${ }^{3,4}$ - F. Petricca ${ }^{1}$ - W. Potzel ${ }^{4} \cdot$ P. Povinec $^{2} \cdot$ F. Pröbst ${ }^{1} \cdot$ F. Reindl $^{6,7}$. J. Rothe ${ }^{1} \cdot$ K. Schäffner ${ }^{1} \cdot$ J. Schieck ${ }^{6,7} \cdot$ V. Schipperges ${ }^{5} \cdot$ D. Schmiedmayer ${ }^{6,7}$. S. Schönert ${ }^{4} \cdot$ C. Schwertner ${ }^{6,7} \cdot$ M. Stahlberg ${ }^{6,7} \cdot$ L. Stodolsky $^{1}$. C. Strandhagen ${ }^{5} \cdot$ R. Strauss ${ }^{4} \cdot$ C. Türkoğlu $u^{6,7} \cdot$ I. Usherov $^{5} \cdot$ M. Willers $^{4}$. V. Zema ${ }^{3,11,12} \cdot \mathrm{J}$. Zeman ${ }^{2} \cdot$ The CRESST Collaboration

Received: 19 August 2019 / Accepted: 13 January 2020 / Published online: 1 February 2020 (c) The Author(s) 2020

\begin{abstract}
Cryogenic Rare Event Search with Superconducting Thermometers (CRESST) is a long-standing direct dark matter detection experiment with cryogenic detectors located at the underground facility Laboratori Nazionali del Gran Sasso in Italy. CRESST-III, the third generation of CRESST, was specifically designed to have a world-leading sensitivity for low-mass dark matter (DM) (less than $2 \mathrm{GeV} / \mathrm{c}^{2}$ ) to probe the spin-independent DM-nucleus cross section. At present, a large part of the parameter space for spin-independent scattering off nuclei remains untested for dark matter particles with masses below few $\mathrm{GeV} / \mathrm{c}^{2}$ although many motivated theoretical models having been proposed. The CRESST-III experiment employs scintillating $\mathrm{CaWO}_{4}$ crystals of $\sim 25 \mathrm{~g}$ as target material for dark matter interactions operated as cryogenic scintillating calorimeters at $\sim 10 \mathrm{mK}$. CRESST-III first data taking was successfully completed in 2018, achieving an unprecedented energy threshold for nuclear recoils. This result extended the present sensitivity to DM particles as light as $\sim 160 \mathrm{MeV} / \mathrm{c}^{2}$. In this paper, an overview of the CRESST-III detectors and results will be presented.
\end{abstract}

Keywords Dark matter $\cdot$ Cryogenic detectors $\cdot$ Rare event searches $\cdot$ Particle identification

M. Mancuso

mancuso@mpp.mpg.de

Extended author information available on the last page of the article 


\section{Introduction}

Dark matter (DM) is one of the most exciting puzzles of modern astro-particle physics. It has been studied for decades, but the nature of this exotic kind of matter is still under investigation. Many cosmological observations prove unambiguously the existence of a non-luminous non-baryonic type of matter of which we can measure the gravitational effects. Among the most convincing observations are galaxies rotation curves [1], gravitational lensing [2] and studies on cosmic microwave background which precisely determined the DM abundance $[3,4]$ in the universe at 5.35 times the total mass of the visible matter. One of the most accredited candidates for DM is weakly interacting massive particles (WIMPs) [5] with masses around the weak scale $\sim 10 \mathrm{GeV}-1 \mathrm{TeV}$. Apart from the "WIMP miracle" [6], other plausible production mechanisms are invoked in dark matter scenarios involving lighter and heavier particles [7-9] ( $10 \mathrm{MeV}-100 \mathrm{TeV})$. Recently, a rising interest points towards low-mass DM models, but a large part of the parameter space for spin-independent scattering off nuclei remains untested for dark matter particles with masses below few $\mathrm{GeV} / \mathrm{c}^{2}$.

CRESST adopts the experimental approach of direct detection, which looks for scattering off nuclei of hypothetical dark matter particles inside a target of ordinary matter. Since the expected recoil spectrum of dark matter particlenucleus elastic scattering shows an exponential rise towards low energies, a small threshold enhances sensitivity and is necessary to investigate low-mass DM. The energy threshold needed to explore DM masses below $\sim 2 \mathrm{GeV} / \mathrm{c}^{2}$ is presently only reachable using cryogenics detectors [10-12]. After the 2015 CRESST-II result [13], the R\&D activity mainly focused on threshold improvement. Geometry and sensor design have been optimised to enhance the sensitivity to low energetic nuclear recoils [14]. The new detector was successfully studied in CRESSTIII. First data taking was completed in 2018 achieving an unprecedented energy threshold for nuclear recoils. In this paper, we introduce the CRESST set-up and detectors, and a short report of the main results of CRESST-III is presented.

\section{Set-Up and Data Processing}

\subsection{Cryogenic Set-Up}

Due to the low event rate from dark matter particle-nucleus elastic scattering, events caused by radioactive background would overcome the signal expected from dark matter particles if not suppressed. To ensure an extremely low background environment, the detector is located at the underground facility Laboratori Nazionali del Gran Sasso in Italy. The deep underground site provides a coverage of at least $1400 \mathrm{~m}$ of rock to shield against cosmic radiation. At the same time, environmental radioactivity induced by the natural long-living isotopes in the surroundings is suppressed as much as possible by multiple layers of passive 
shielding. These are composed of $14 \mathrm{~cm}$ of radiopure copper which directly surrounds the experimental volume, followed by $20 \mathrm{~cm}$ of lead with a low ${ }^{210} \mathrm{~Pb}$ activity of $35 \mathrm{~Bq} / \mathrm{kg}$. A $50-\mathrm{cm}$-thick polyethylene is placed outside the $\mathrm{Cu}$ and $\mathrm{Pb}$ shields as a neutron moderator.

To avoid contamination of ${ }^{220} \mathrm{Rn}$ and ${ }^{222} \mathrm{Rn}$, the atmosphere inside the entire shielding is constantly flushed with pure nitrogen gas. The highest external background contribution is the remaining neutron flux, which is dominated by muoninduced neutrons in the lead shield. This eventuality is prevented by the muon veto system which surrounds the entire set-up and by a 5 -cm-thick polyethylene layer inside the $\mathrm{Pb} / \mathrm{Cu}$ shields.

\subsection{Detector Module}

In the first data taking of CRESST-III, an array of 10 scintillating $\mathrm{CaWO}_{4}$ crystals is read out simultaneously as cryogenic calorimeters and scintillating detectors. Despite the effort to shield the set-up, the unavoidable natural radioactivity of its materials would be enough to shade a DM signal. The double read-out provides a tool for particle identification on an event-by-event basis, and it is used for suppressing background induced by charged particles [15], while the heat signal provides a precise measurement of the total energy deposition, independently of the type of particle interaction. This feature, in combination with a low energy threshold, makes cryogenic calorimeters particularly suited for low-mass dark matter detection.

Each detector consists of a $(20 \times 20 \times 10) \mathrm{mm}^{3} \mathrm{CaWO}_{4}$ scintillating crystal of $\sim 25 \mathrm{~g}$ and a second smaller nearby cryogenic calorimeter made of silicon-onsapphire (SOS) for the detection of the scintillating light. This detector concept is thoroughly discussed in [16]. Both calorimeters are equipped with transition edge sensors (TESs) to measure energy deposition which are designed to provide a threshold of $\leq 100 \mathrm{eV}$ for the $\mathrm{CaWO}_{4}$ crystals and $\sim 20 \mathrm{eV}$ for the silicon-on-sapphire detectors.

In addition, CRESST-III detectors are also equipped with a fully scintillating housing and instrumented holders to veto the possible background radiation originating from the surrounding surfaces. This innovative type of active holding system allows to suppress induced thermal signals from particle interaction in the holding materials-see Fig. 1.

Each detector is equipped with a specially designed TES to optimise the phonon collection (i.e. the signal amplitude). In particular, the $\mathrm{CaWO}_{4}$ crystal has a tungsten film of $(2.4 \times 0.85) \mathrm{mm}^{2}$ and $200 \mu \mathrm{m}$ thick weakly coupled to the heat bath by a thermal link of about $100 \mathrm{pW} / \mathrm{K}$ at $10 \mathrm{mK}$. The thermal coupling is realised by a Au stripe $(1.0 \times 0.02) \mathrm{mm}^{2}$ and $20 \mathrm{~nm}$ thick. Similarly, the light detector is equipped with a TES which is $~ 100$ times smaller. Next to the TES sensors, an heater element made by a thin strip of $\mathrm{Au}$ is placed, to which a pattern of current pulses is periodically delivered, to simulate thermal pulses in the detector response. The use of test pulses allows stabilisation and monitoring of the detector response over time. TESs with a light detector design are evaporated on Si carriers $(3 \times 3.5 \times 0.4) \mathrm{mm}^{3}$ 

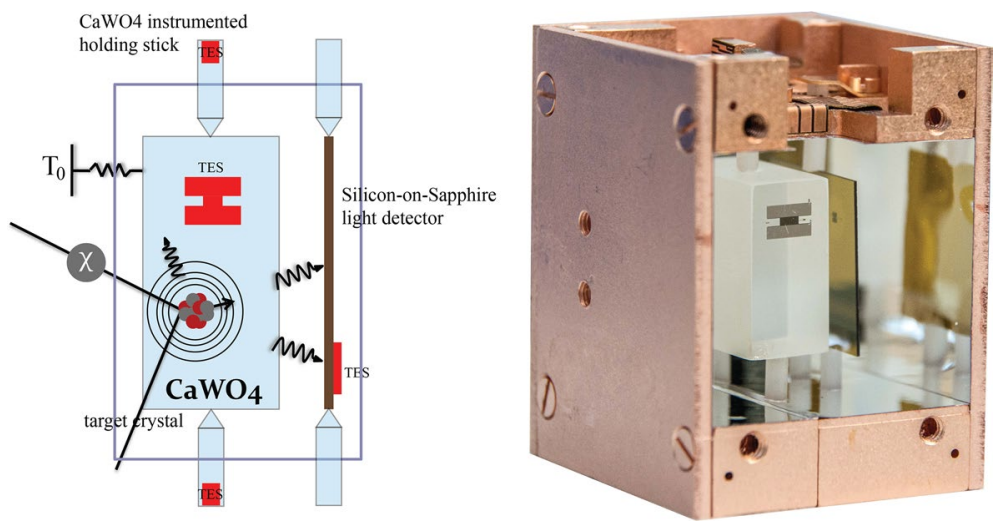

Fig. 1 Left: schematic view of the detector design for CRESST-III modules. A DM particle scattering off a nucleus produces phonons, measured by the TES deposited on the target crystal, and photons, collected by the SOS light detector. Both detectors are weakly coupled to a thermal bath at $T_{0} \sim 10 \mathrm{mK}$. A fully scintillating housing and instrumented $\mathrm{CaWO}_{4}$ holder sticks are used to veto a possible background originating from surrounding surfaces. Right: picture of a CRESST-III detector module (Color figure online)

which are glued onto the $\mathrm{CaWO}_{4}$ sticks holding the target crystal. For further details, see [17].

\subsection{Data Acquisition}

In CRESST-III, the detectors output is continuously recorded with a sampling rate of $25 \mathrm{kHz}$ with a dead-time free transient digitizers. The full recorded signal is filtered with an offline software trigger which uses a dedicated matched filter adapted to each detector to maximise the signal-to-noise ratio. The transfer function used was the so-called optimum filter transfer function [18], $H(j \omega)$ which is built using only the noise power spectrum $\mathscr{N}(\omega)$ and the response function $s(t)$ of the detector after a particle interaction (set as an averaged particle pulse). When the software trigger identifies an event for each channel, we select a record window $655.36 \mathrm{~ms}$ for further analysis on pulse shape parameters. Since the output of the optimum filter maximises the signal-to-noise ratio, it is not only used for the software triggering, but also as basis for the energy reconstruction. The method and the threshold selection criteria adopted are thoroughly discussed in [19].

\subsection{Calibration and Energy Threshold}

During the data taking reported here, two calibration campaigns have been carried out: one used an external source of ${ }^{57} \mathrm{Co}$ to asses the energy response, while the other used an AmBe source for neutron calibration. The gamma source ${ }^{57} \mathrm{Co}$ produces two mono-energetic lines capable of penetrating the cryostat's inner shields, one at $122 \mathrm{keV}$ and one at $136 \mathrm{keV}$. Given the high sensitivity of the detectors at $\sim 100 \mathrm{keV}$, the detector response is completely saturated, which means that the 

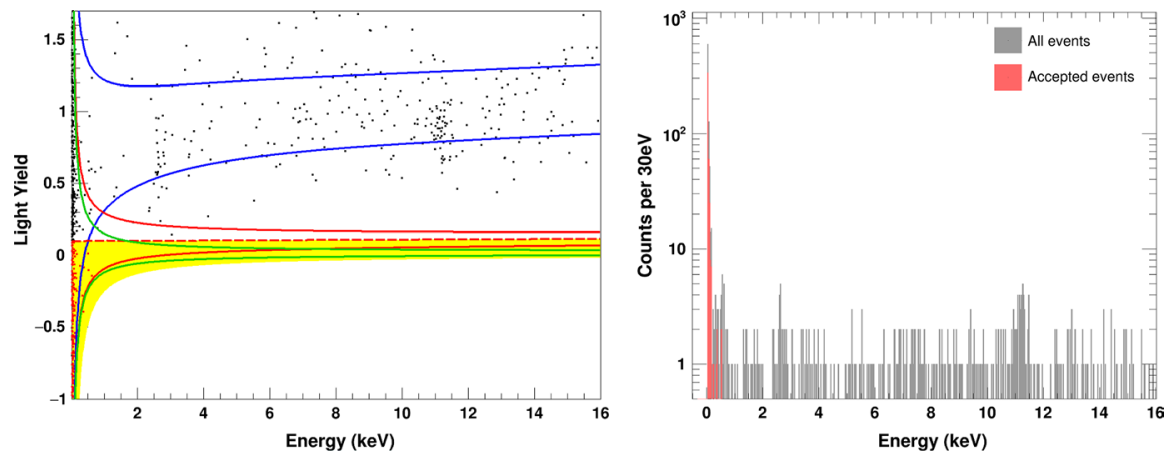

Fig. 2 Left: light yield versus energy of events in the dark matter data set. The blue band represents $90 \%$ of the upper and lower boundaries of the $\beta / \gamma$-band while the red and green bands represent oxygen and tungsten, respectively. The yellow shaded area denotes the acceptance region reaching from the mean of the oxygen band (red dashed line) down to the $99.5 \%$ lower boundary of the tungsten band. Events in the acceptance region are highlighted in red. The position of the bands is extracted from the neutron calibration data. Right: energy spectrum of the dark matter data set with lines visible at $2.6 \mathrm{keV}$ and $11.27 \mathrm{keV}$ originating from cosmogenic activation of ${ }^{182} \mathrm{~W}$. Gray: all events, red: events in the acceptance region (Color figure online)

TES reaches the normal conducting state. Therefore, an initial calibration was made using the escape peak from $K_{\alpha 1}$ and $K_{\alpha 2}$ of $W$, resulting in a line of $63.2 \mathrm{keV}$. Later the calibration was adjusted with another mono-energetic line at $11.27 \mathrm{keV}$ present in the data due to a cosmogenic activation of tungsten (see Fig. 2).

The neutron source is used to calibrate the light yield, defined as $\mathrm{LY}=E_{\mathrm{l}} / E_{\mathrm{ph}}$, where $E_{1}$ is the energy estimator of the light detector response and $E_{\mathrm{ph}}$ is the energy estimator of the phonon channel which is considered to be the total deposited energy of a particle interaction, $\mathrm{LY} \equiv 1$ for electron and gamma interaction. The induced neutron interactions are used to fit the bands of nuclear recoils for every detector module: The band description we used is presented in [20]. In Fig. 2(left), the red and green lines mark the bands expected for recoils off oxygen and tungsten, respectively. The calcium band lies between the oxygen and the tungsten bands and is not drawn for clarity. The bands are fitted with an unbinned maximum likelihood approach. Figure 2(right) shows the background data set of one detector module (named detector A) which corresponds to an exposure before cuts of $5.689 \mathrm{~kg}$ day. More details about the analysis and the data selection can be found in [12].

\section{Dark Matter Results}

The accepted events for dark matter analysis are shown in Fig. 2 marked in red on the right. Below $\sim 200 \mathrm{eV}$, the event rate rises and appears to be exponentially leaning towards low energy. The source of these events is not understood [12] and still under investigation. The trigger and analysis threshold of the detector module used for DM analysis have been set at $30.1 \mathrm{eV}$. The expected triggers in noise 
contribute for 1 count/(kg days) according to [19]. The LY parameter does not allow for particle discrimination at this energy scale; therefore, it is not possible to determine whether this background is caused by nuclear recoils or $\beta / \gamma$ interactions (see Fig. 2(left)). This rise of events is measured by all CRESST-III detector modules with thresholds below $100 \mathrm{eV}$ : The energy spectra of these events vary for different modules, which argues against a single common source.

To calculate the limit on spin-independent and spin-dependent interactions of dark matter particles with nuclei, reported in [12], all the measured events have been considered as potential signal events, not performing any background subtraction. The result is reprinted in Fig. 3. The Yellin's optimal interval method $[21,22]$ has been used, and the standard dark matter halo model used has the following properties: dark matter halo with a Maxwellian velocity distribution, a local dark matter density of $\rho_{\mathrm{DM}}=0.3 \mathrm{GeV} /\left(\mathrm{c}^{2} \mathrm{~cm}^{3}\right)$ [23], the galactic escape velocity of $v_{\mathrm{esc}}=544 \mathrm{~km} / \mathrm{s}$ [24] and $v_{\odot}=220 \mathrm{~km} / \mathrm{s}$ for the solar orbit velocity [25].

The spin-dependent exclusion limit (Fig. 3(right)) is calculated accounting for the natural presence of the isotope ${ }^{17} \mathrm{O}$ which is sensitive to spin-dependent
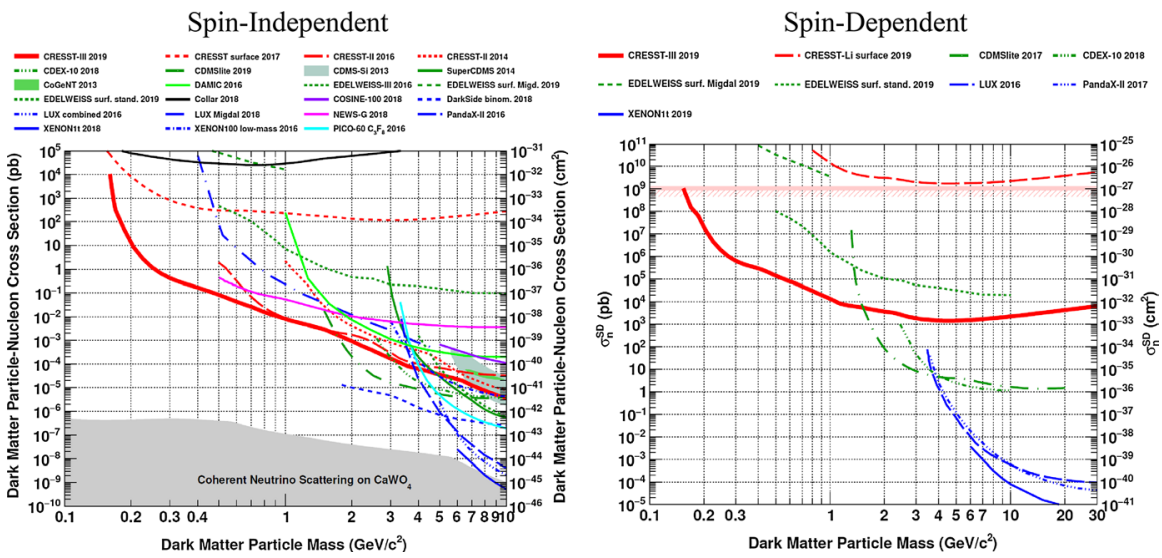

Fig. 3 Left: experimental results on elastic, spin-independent dark matter nucleus scattering depicted in the cross section versus dark matter particle mass. Results are reported with $90 \%$ confidence level (CL). The result of this work in solid red with the most stringent limit between masses of (0.16-1.8) GeV/c2. The previous CRESST-II result in dashed red [13], the red dotted line corresponds to a surface measurement performed with a gram-scale $\mathrm{Al}_{2} \mathrm{O}_{3}$ detector [29]. A colour coding to group the experimental results is used: Green for exclusion limits (CDEX [30], CDMSlite [31], DAMIC [32], EDELWEISS [33, 34], SuperCDMS [35]) and positive evidence (CDMS-Si (90\% CL) [35], CoGeNT (99\% CL) [36]) obtained with solid-state detectors based on silicon or germanium, blue for liquid noble gas experiments based on argon or xenon (DarkSide [37], LUX [38, 39], Panda-X [40], Xenon100 [41], Xenon1t [42]), violet for COSINE-100 (NaI) [43], black for Collar (H) [44], magenta for the gaseous spherical proportional counter NEWS-G (Ne + CH4) [45] and cyan for the superheated bubble chamber experiment PICO (C3F8) [46]. The gray region marks the so-called neutrino floor calculated for CaWO4 in [47]. Right: results on spin-dependent neutron-only interactions via the isotope ${ }^{17} \mathrm{O}$ in solid red (this work) and a result with ${ }^{7} \mathrm{Li}$ in dashed red [26]. Additionally, we plot results from CDMSlite on ${ }^{73} \mathrm{Ge}$ [48], LUX [49], Panda-X [50] and XENON1t [51], all three on ${ }^{129} \mathrm{Xe}$ and ${ }^{131} \mathrm{Xe}$. Figure reprinted from [12] (Color figure online) 
neutron-only interactions. The expected rate for spin-dependent interaction follows [26] but is corrected for the isotope properties: nuclear spin $(J=+5 / 2)$, the mass $(A=17)$ and the spin matrix element $\left(\left\langle S_{\mathrm{n}}\right\rangle=0.5\right)[27,28]$. Accounting for the natural abundance, the gross exposure of ${ }^{17} \mathrm{O}$ is $0.46 \mathrm{~g}$ days.

\section{Conclusion and Perspective}

The new limit obtained with CRESST-III [12] confirms and extends the results of the previous generation of CRESST (red solid line vs red dashed line in Fig. 3). The lower energy threshold allowed to improve the reach by one order of magnitude in cross section for DM particles with mass of $0.5 \mathrm{GeV} / \mathrm{c}^{2}$ and extends the sensitivity range of DM mass down to $160 \mathrm{MeV} / \mathrm{c}^{2}$ compared to the previous CRESST results [13]. The observation of a rising event rate, which is incompatible with a flat background assumption, limits the sensitivity to DM interaction. The understanding of the background becomes new focus of the experiment. A second data taking with CRESST-III is mainly devoted to hardware tests with upgraded detector modules to study the origins of low energy events.

Acknowledgements Open Access funding provided by Projekt DEAL.

Open Access This article is licensed under a Creative Commons Attribution 4.0 International License, which permits use, sharing, adaptation, distribution and reproduction in any medium or format, as long as you give appropriate credit to the original author(s) and the source, provide a link to the Creative Commons licence, and indicate if changes were made. The images or other third party material in this article are included in the article's Creative Commons licence, unless indicated otherwise in a credit line to the material. If material is not included in the article's Creative Commons licence and your intended use is not permitted by statutory regulation or exceeds the permitted use, you will need to obtain permission directly from the copyright holder. To view a copy of this licence, visit http://creativecommons.org/licen ses/by/4.0/.

\section{References}

1. V.C. Rubin, N. Thonnard, W.K. Ford Jr., Astrophys. J. 238, 471 (1980)

2. D. Clowe, M. Bradac, A.H. Gonzalez, M. Markevitch, S.W. Randall, C. Jones, D. Zaritsky, Astrophys. J. 648, L109-L113 (2006). https://doi.org/10.1086/508162

3. Planck Collaboration, P. A. R. Ade et al., Planck 2013 results. XVI. Cosmological parameters, Astron. Astrophys.571 (2014) A16. https://doi.org/10.1051/0004-6361/201525830

4. Planck Collaboration, P. A. R. Ade et al., Planck 2013 results. XVI. Cosmological parameters, Astron. Astrophys.571 (2014) A16. https://doi.org/10.1051/0004-6361/201321591

5. G. Steigman, M.S. Turner, Nuclear Phys. B 253, 375-386 (1985). https://doi.org/10.1016/05503213(85)90537-1

6. G. Jungman, M. Kamionkowski, K. Griest, Phys. Rep. 267, 195-373 (1996). https://doi. org/10.1016/0370-1573(95)00058-5

7. D.E. Kaplan, M.A. Luty, K.M. Zurek, Phys. Rev. D 79, 115016 (2009). https://doi.org/10.1103/ PhysRevD.79.115016

8. C. Boehm, P. Fayet, Nucl. Phys. B 683, 17 (2004). https://doi.org/10.1016/j.nuclp hysb.2004.01.015

9. G. Bertone, D. Hooper, J. Silk, Phys. Rep. 405, 279 (2005) 
10. SuperCDMS Collaboration (R. Agnese (Florida U.) et al.). Phys. Rev. Lett. 121 (2018). https://doi. org/10.1103/PhysRevLett.122.069901

11. EDELWEISS Collaboration (Q. Arnaud (Lyon, IPN) et al.). Phys. Rev. D 97 (2018). https://doi. org/10.1103/PhysRevD.97.022003

12. The CRESST collaboration, Astroparticle Physics. Mar 31, 2019. arXiv: 1904.00498

13. G. Angloher et al. (CRESST) 2016 Eur. Phys. C.C76 25 (Preprint 1509.01515)

14. G. Angloher et al. (CRESST) 2015 (Preprint 1503.08065)

15. R. Strauss et al., CRESST. NIM A 845, 414-417 (2017). https://doi.org/10.1016/j.nima.2016.06.060

16. R. Strauss et al. (CRESST) 2015 Eur. Phys. J.C75, 352 (Preprint 1410.1753)

17. M. Mancuso, G. Angloher, P. Bauer et al., J. Low Temp. Phys. 193, 441-448 (2018). https://doi. org/10.1007/s10909-018-1948-6

18. E. Gatti, P.F. Manfredi, Il Nuovo Cimento 9, 549 (1986)

19. M. Mancuso et al., NIM A 940, 492-496 (2019). https://doi.org/10.1016/j.nima.2019.06.030

20. R. Strauss et al., Eur. Phys. J. C 74, 1 (2014)

21. S. Yellin, Phys. Rev. D 66, 032005 (2002)

22. S. Yellin, Software for computing an upper limit given unknown background (02/2011). http://cdms. stanford.edu/Upperlimit/

23. P. Salucci et al., Astron. Astrophys. 523, 9 (2010). https://doi.org/10.1051/0004-6361/201014385

24. M.C. Smith et al., Mon. Not. R. Astron. Soc. (2007). https://doi.org/10.1111/j.1365-2966.2007.11964.x

25. F.J. Kerr, D. Lynden-Bell, Mon. Not. R. Astron. Soc. (1986). https://doi.org/10.1093/mnras /221.4.1023

26. The CRESST collaboration, Eur. Phys. J. C (2019). https://doi.org/10.1140/epjc/s10052-019-7126-4

27. V.A. Bednyakov, F. Simkovic, Phys. Part. Nucl. 36, 131-152 (2005)

28. A.F. Pacheco, D. Strottman, Phys. Rev. D 40, 2131 (1989)

29. G. Angloher et al., Eur. Phys. J. C 77, 637 (2017)

30. C.D.E.X. Collaboration et al., Phys. Rev. Lett. 120, 241301 (2018)

31. SuperCDMS Collaboration et al., Phys. Rev. D99, 062001 (2019)

32. DAMIC Collaboration et al., Phys. Rev. D94, 082006 (2016)

33. L. Hehn et al., Eur. Phys. J. C 76, 107 (2016)

34. EDELWEISS Collaboration et al., Phys. Rev. D99, 082003 (2019)

35. SuperCDMS Collaboration et al., Phys. Rev. Lett.112, 051301 (2014)

36. CoGeNT Collaboration et al., Phys. Rev. D88, 012002 (2013)

37. DarkSide Collaboration et al., Phys. Rev. Lett.121, 111303 (2018)

38. L.U.X. Collaboration et al., Phys. Rev. Lett. 118, 251302 (2017)

39. L.U.X. Collaboration et al., Phys. Rev. Lett. 122, 131301 (2019)

40. PandaX-II Collaboration et al., Phys. Rev. Lett.119, 181302 (2017)

41. XENON Collaboration et al., Phys. Rev. D94, 122001 (2016)

42. XENON Collaboration et al., Phys. Rev. Lett.121, 111302 (2018)

43. COSINE-100 Collaboration et al., Nature564, 83 (2018)

44. J. Collar, Phys. Rev. D 98, 023005 (2018)

45. Q. Arnaud et al., Astropart. Phys. 97, 54 (2018)

46. P.I.C.O. Collaboration et al., Phys. Rev. D 93, 052014 (2016)

47. A. Gütlein et al., Astropart. Phys. 69, 44 (2015)

48. S.C.D.M.S. Collaboration et al., Phys. Rev. D 97, 022002 (2018)

49. L.U.X. Collaboration et al., Phys. Rev. Lett. 116, 161302 (2016)

50. PandaX-II Collaboration et al., Phys. Rev. Lett. 118, 071301 (2017)

51. E. Aprile et al., arXiv:1902.03234 (2019)

Publisher's Note Springer Nature remains neutral with regard to jurisdictional claims in published maps and institutional affiliations. 


\section{Affiliations}

M. Mancuso ${ }^{1}$ - A. H. Abdelhameed ${ }^{1} \cdot$ G. Angloher ${ }^{1} \cdot$ R. Breier $^{2}$ - P. Bauer ${ }^{1}$. A. Bento ${ }^{1,10} \cdot$ E. Bertoldo $^{1}$. C. Bucci ${ }^{3} \cdot$ L. Canonica $^{1}$ - A. D'Addabbo ${ }^{3,11}$. S. Di Lorenzo ${ }^{3,11} \cdot$ A. Erb $^{4,9} \cdot$ F. von Feilitzsch ${ }^{4} \cdot$ N. Ferreiro lachellini ${ }^{1}$. S. Fichtinger ${ }^{6} \cdot$ A. Fuss ${ }^{6,7} \cdot$ P. Gorla ${ }^{3} \cdot$ D. Hauff ${ }^{1} \cdot$ M. Ješkovský ${ }^{2} \cdot$ J. Jochum $^{5}$. J. Kaizer ${ }^{2} \cdot$ A. Kinast ${ }^{4} \cdot$ H. Kluck ${ }^{6,7} \cdot$ H. Kraus ${ }^{8} \cdot$ A. Langenkämper ${ }^{4}$. V. Mokina ${ }^{6}$. E. Mondragon ${ }^{4} \cdot \mathrm{M}$. Olmi ${ }^{3,11} \cdot$ T. Ortmann $^{4} \cdot$ C. Pagliarone ${ }^{3,13} \cdot$ V. Palušová ${ }^{2}$. L. Pattavina ${ }^{3,4} \cdot$ F. Petricca ${ }^{1} \cdot$ W. Potzel ${ }^{4} \cdot$ P. Povinec ${ }^{2} \cdot$ F. Pröbst ${ }^{1} \cdot$ F. Reind ${ }^{6,7}$. J. Rothe ${ }^{1} \cdot$ K. Schäffner ${ }^{1} \cdot$ J. Schieck ${ }^{6,7} \cdot$ V. Schipperges ${ }^{5} \cdot$ D. Schmiedmayer ${ }^{6,7}$. S. Schönert ${ }^{4} \cdot$ C. Schwertner ${ }^{6,7} \cdot$ M. Stahlberg ${ }^{6,7} \cdot$ L. Stodolsky $^{1}$.

C. Strandhagen ${ }^{5} \cdot$ R. Strauss ${ }^{4} \cdot$ C. Türkoğlu ${ }^{6,7} \cdot$ I. Usherov ${ }^{5} \cdot$ M. Willers ${ }^{4}$. V. Zema ${ }^{3,11,12} \cdot$ J. Zeman ${ }^{2} \cdot$ The CRESST Collaboration

1 Max-Planck-Institut für Physik, 80805 Munich, Germany

2 Faculty of Mathematics, Physics and Informatics, Comenius University, 84248 Bratislava, Slovakia

3 Laboratori Nazionali del Gran Sasso, INFN, 67100 Assergi, Italy

4 Physik-Department and Excellence Cluster Universe, Technische Universität München, 85748 Garching, Germany

5 Eberhard-Karls-Universität Tübingen, 72076 Tübingen, Germany

6 Institut für Hochenergiephysik der Österreichischen Akademie der Wissenschaften, 1050 Wien, Austria

7 Atominstitut, Vienna University of Technology, 1020 Wien, Austria

8 Department of Physics, University of Oxford, Oxford OX1 3RH, UK

9 Walther-Meißner-Institut für Tieftemperaturforschung, 85748 Garching, Germany

10 Departamento de Fisica, Universidade de Coimbra, 3004516 Coimbra, Portugal

11 GSSI-Gran Sasso Science Institute, 67100 L'Aquila, Italy

12 Department of Physics, Chalmers University of Technology, 41296 Göteborg, Sweden

13 Dipartimento di Ingegneria Civile e Meccanica, Università degli Studi di Cassino e del Lazio Meridionale, 03043 Cassino, Italy 